\title{
Comparing Verification Performance of Kids and Adults for Fingerprint, Palmprint, Hand-geometry and Digitprint Biometrics.
}

\author{
Andreas Uhl and Peter Wild
}

\begin{abstract}
With the large scale deployment of biometrics for access control in private and public places, systems are faced the challenge of processing a diverse range of people. Most systems have been well evaluated for adults, however, their application in schools, or for private door access control, raises the question, whether there exists significant difference in performance between age groups in general and between kids and adults in particular. This paper targets an evaluation of the impact of children as biometric users on recognition accuracy for a series of hand-based modalities: Fingerprint, Palmprint, Hand-geometry and Digitprint. Furthermore, we try to analyze reasons for child-aging effects on performance at both feature and instance level using our database of 301 kids and 86 adults.
\end{abstract}

\section{INTRODUCTION}

Biometric system performance based on child data is not well reported in literature. Even for studies devoted to assessing the impact of age on feature quality [1], [2], the age group of people under 18 years has been largely neglected so far. However, the use of biometric systems for kids becomes more and more an important issue, since many advantages of biometrics are even more pronounced for the young generation [3]: (a) biometrics may neither easily be lost without intention, nor forgotten like passwords; (b) biometric systems enable large throughput for access control, which is an important factor when applied in e.g. schools; and (c) holding tokens or passwords does not necessarily imply legal ownership, both theft and fraudulent passing of tokens (such as entry tickets) or knowledge can be avoided. Because of these and further reasons, biometric systems are being increasingly adopted for applications where kids are at least a part of the target user group. Fidelity of a biometric system, which represents how closely samples from the same individual match, might largely be affected by age. The purpose of this work is to estimate and quantify differences in performance of the observed modalities, when applied to palmar hand images of children instead of adults.

The most frequently applied biometric modality according to the Biometric Market Report conducted by the International Biometric Group is certainly the Fingerprint modality. However, we do not restrict our survey to the impact of infancy on recognition performance of fingerprints, but assess several hand-based modalities because of the following reasons: (a) recently the successful combination of fingerprints, palmprints and hand geometry from single palmar images of the human hand has been shown to increase recognition rates

A. Uhl and P. Wild are with the Department of Computer Sciences, University of Salzburg, A-5020 Salzburg, Austria \{uhl, pwild\}@cosy.sbg.ac.at significantly [4], [5], an idea which is likely to be exploited by future systems; and (b) Palmprint and Hand-geometrybased modalities have itself a strong market presence.

By assessing the impact of child age on recognition accuracy, we extend previous work by Modi et al. [1], [2], who compared different adult age groups in terms of fingerprint quality - and therefore performance. According to [1], error rates are typically increased in fingerprint systems, when deployed for use by subjects from a senior (62 years or older) age group. This primary study is extended in [2], showing, that fingerprint image quality for four age groups (18-25, 26$39,40-62$, and $62+)$ is significantly different and degrading (most pronounced with the $62+$ age group). A further result in their work is the overall quality score degradation with increasing variances as the age increases. As possible reasons for the observed behaviour, decreased skin firmness (aging due to loss of collagen) and medical sufferings (arthritis) are given. Some of the acquisition problems, such as nonuniform and irreproducible contact may be characteristic for specific age groups, i.e. we expect a negative influence on recognition accuracy not only by very old users as reported in [2], but also for very young users. Of course, not all factors may be reduced to age, such as e.g. injuries, disease, scars, or genetical abnormalities as e.g. dermathopatia pigmentosa causing a lack of fingerprints.

While quality metrics are certainly a good measure to predict performance, we directly assess the impact on matching scores in this work. We also focus on a comparison of different hand regions with respect to their resistance to aging affects for kids. Since this may also depend on selected features (e.g. [6] show an increased impact of quality degradation to minutiae-based matchers compared to ridge-based fingerprint matchers), we also look at different feature extraction algorithms related to the human hand as a biometric trait. Main contributions of this work comprise: (a) a comparison of verification performance of kids and adults for Fingerprint, Palmprint, Hand-geometry and Digitprint biometric modalities; (b) an assessment of performance differences between different child age groups (3-10, 11-18); (c) an intra-feature comparison of age impact on different instances, i.e. regions of interest (thumb, index, middle, ring, little finger and palm); and (d) an analysis of possible reasons for performance differences and similarities.

This paper is structured as follows: the employed test database of child and adult images is introduced in Sect. II. Section III presents the employed biometric system including an overview of implemented preprocessing methods to extract and enhance single hand regions, as well as feature

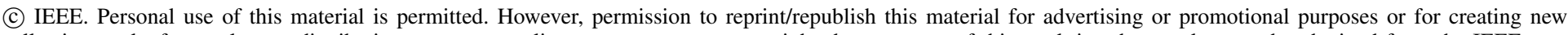

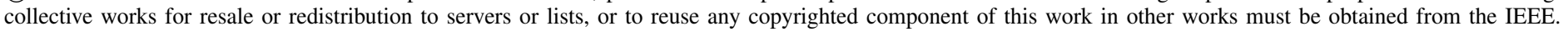

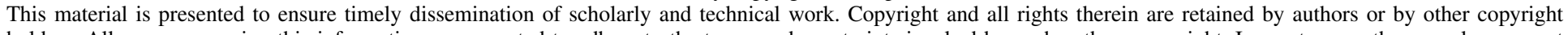

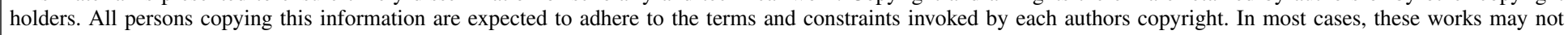
be reposted without the explicit permission of the copyright holder. 
TABLE I

Distribution OF AGES 3 - 18 IN THE DATASET

\begin{tabular}{|c||c|c|c|c|c|c|c|c|}
\hline Age & 3 & 4 & 5 & 6 & 7 & 8 & 9 & 10 \\
\hline \hline Users & 1 & 0 & 10 & 10 & 19 & 26 & 37 & 42 \\
\hline Templates & 1 & 0 & 16 & 17 & 35 & 45 & 66 & 69 \\
\hline
\end{tabular}

\begin{tabular}{|c||c|c|c|c|c|c|c|c|}
\hline Age & 11 & 12 & 13 & 14 & 15 & 16 & 17 & 18 \\
\hline \hline Users & 49 & 51 & 16 & 18 & 15 & 6 & 0 & 1 \\
\hline Templates & 84 & 72 & 29 & 29 & 25 & 9 & 0 & 1 \\
\hline
\end{tabular}

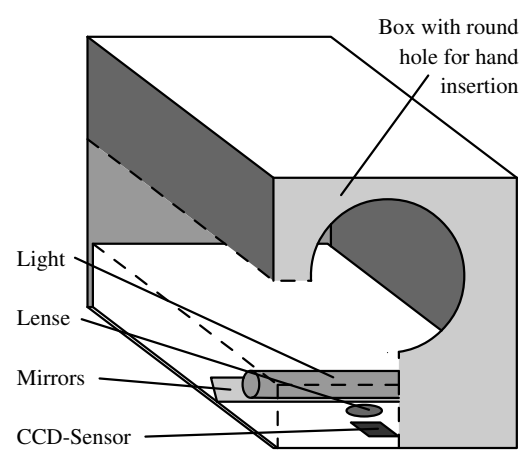

Fig. 1. Employed flatbed scanner solution with containment.

extraction and matching algorithms. Performance results comparing kids and adults are discussed in Sect. IV. Finally, Sect. V forms the conclusion.

\section{DATABASE}

We collected our database in two phases, starting with a three-day capture of adult hands by volunteers (mainly students) aged above 18 at our university. This dataset of 86 users and 443 templates has been reduced to 172 templates by taking the first and last acquired template for each user in order to conduct fair comparisons with respect to the number of genuine comparisons and time span issues. Furthermore, a separate small dataset of adults has been recorded for training purposes (i.e. for the calculation of Eigenfingers and Eigenpalms).

Data for the young age groups (18 or less years) were collected on three days at an event called Uni:Hautnah, where a public audience (mainly children at school age) gets in touch with local research conducted at the University of Salzburg by visiting research booths in a shopping center. After single enrollment, users could re-visit the booth on the same day at any time (a minimum time lapse of 30 minutes was encouraged) for identification. A total number of 498 images of 301 young volunteers aged from 3 to 18 were acquired, see Tab. I (some users did not re-visit the booth, very few re-visited the booth more than once).

Both data series were recorded with the same optical scanning device, a HP 3500c flatbed scanner recording a $216 \times 297$ millimeters image at $500 \mathrm{dpi}$. For image acquisition, the scanner is contained in a box in order to control environmental light, see Fig. 1. While this scanner is able to acquire FBI-recommended 500 dpi resolution, still acquisition time is rather high with this model, i.e. 95 seconds on average (this can be reduced with newer models to, e.g., 43 sec. using HP G3010, see [5]).

\section{SYSTEM}

In order to evaluate the performance of different modalities, we employed a multibiometric hand-based system able to extract minutiae, hand geometry information, palmprint and digitprint features from a single palmar scan of the human hand [5]. Each modality is extracted from its region of interest and matched independently of the other features in the system.

\section{A. Preprocessing}

The employed system is peg-free and allows users to choose an arbitrary placement of the hand on the sensor as long as fingers do not touch each other. In order to obtain rotational and translational invariance, several preprocessing steps are applied, which will be outlined in this section. For a more detailed description of employed algorithms, we refer to [5], [7]. In a first step, the $4250 \times 5850$ input image is segmented using Otsu's thresholding and visible arm parts are removed iteratively using best-ellipse fitting. For the extraction of regions of interest for single features, additional alignment steps are executed. The following regions of interest are extracted: (a) high-resolution fingerprint images (size depends on the actual finger length), (b) digitprint images using best-ellipse fitting of individual fingers, (c) palmprint image located by means of a palm coordinate system centered in the valley between middle and ring fingers.

\section{B. Feature extraction and matching}

We employ five different geometric and texture-based algorithms (see also [7]):

- Minutiae: identifies minutiae points within a finger-axisaligned rectangle at finger tips using NIST's minutiae extraction software [8].

- Eigenpalms and Eigenfingers: projects each finger and palm onto the eigenspace spanned by the most significant principal components (see [9]).

- Geometry: identifies finger parts (proximal, intermediate and distal phalanx) and keeps track of individual length and width (see e.g. [10]).

- Shape: divides each finger into slices of equal height and keeps track of the local finger width for each slice.

- Palmprint: extracts variances of 144 overlapping blocks in the palmprint image after edge detection (method introduced by Kumar et al. [11]).

Information of the corresponding features can be expected to be largely independent, since employed algorithms operate at:

- different resolutions: Shape, Minutiae, Palmprint and Geometry require the full 500 dpi input signal, Eigenpalms and Eigenfingers are extracted at $100 \mathrm{dpi}$.

- different parts of the hand: Minutiae operates on each of the five finger tips, Eigenpalms and Palmprint extract 


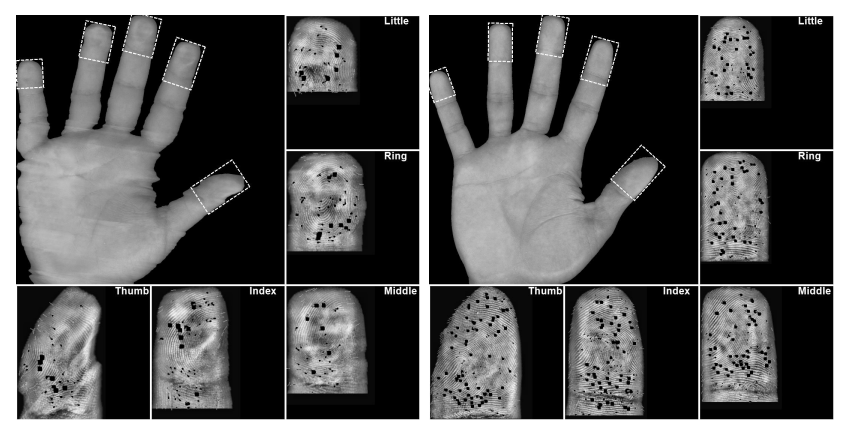

Fig. 2. Fingerprint of 5-year-old boy (left) vs. adult (right).

information from the textural palm area while Eigenfingers, Shape and Geometry concentrate on fingers.

1) Minutiae: We extract position, orientation and quality of termination and bifurcation points in the outermost structural part of the epidermis employing the NFIS2 [8] extraction software mindtct on the enhanced fingerprint image. While sensing regions may also be defined for other parts of the human hand (see [4] for results on metacarpal skin), we extract each fingerprint image as a $w \times \frac{h}{3}$ (and $w \times \frac{h}{2}$ for the thumb, respectively) rectangular area aligned with respect to the major axis of the finger circumscribed by its $w \times h$ sized bounding box. Since flatbed optical images exhibit low contrast between ridges and valleys, ContrastLimited Histogram Equalisation described in [12] has been employed. Minutiae pairing and matching is executed using the NFIS2 bozorth3 matcher. Examples of extracted minutiae from child and adult data are illustrated in Fig. 2.

2) Eigenpalms and Eigenfingers: Eigenpalms and Eigenfingers are derived from Eigenfaces introduced by Turk and Pentland [13] and are based on the Karhunen-Loeve transform converting image vectors of single fingers or palms into a space spanned by the most significant components calculated from a set of training images. After subtracting the average image vector $\mathfrak{a}$ for each type of training image $(256 \times 256$ palm images, $128 \times 384$ index, middle, ring fingers and $128 \times 256$ thumb and little finger images) resulting in normalised samples $\mathfrak{n}_{i}$, the $l=25$ eigenvectors $\mathfrak{u}_{j}$ with largest eigenvalues are computed from covariance matrix $\mathfrak{C}$ :

$$
\mathfrak{C}=\frac{1}{x} \sum_{i=1}^{x} \mathfrak{n}_{i} \mathfrak{n}_{i}^{T}=\mathfrak{A A}^{T}
$$

For each image type feature extraction corresponds to (a) extraction of finger or palm image $\mathfrak{n}$; (b) normalisation with respect to the average image vector $\mathfrak{a}$ and; (c) projection onto the corresponding space spanned by $\mathfrak{u}_{j}$ and storage of projection coefficients as feature components, see Fig. 3. The corresponding matcher is based on Manhattan metric.

3) Geometry feature: This simple geometric feature estimates the length of proximal, intermediate and distal phalanx by searching the normalised finger within certain windows for the vertical slice of height $h=30$ with the lowest median of pixel intensities using the local image histogram. The feature vector is composed of the height of each found finger part and average left and right width of the extracted finger,
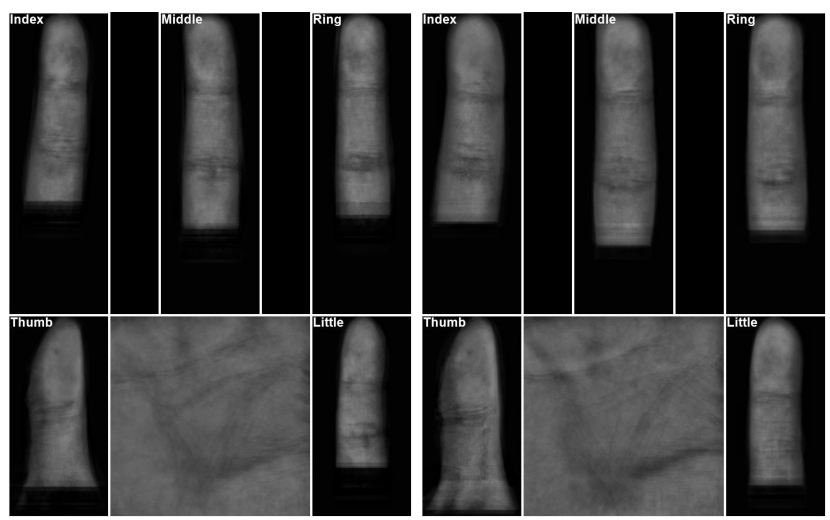

Fig. 3. Eigenfingers and Eigenpalm of 11-year-old girl (left) vs. adult (right).
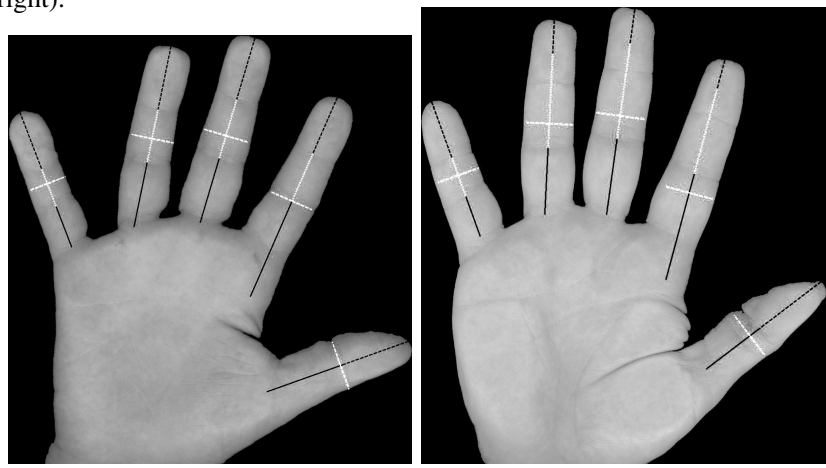

Fig. 4. Geometry feature for 5-year-old boy (left) vs. adult (right).

see Fig. 4. Matching involves the calculation of a simple distance metric.

4) Shape feature: The Shape feature targets hand geometry measurements extracting local finger widths from the hand contour, see Fig. 5. Each finger is rotated upright using moments and cropped at the adjacent finger valley closer to the tip. For each of three slices of equal height covering the finger we determine the object's average width with respect to the y-monotone contour extracted with a left-right scan method. Matching is based on Manhattan metric.

5) Palmprint feature: After localisation of the hand-axisaligned square palmprint region of size $\bar{t}$ (being the average finger length) centered in $P=(0,-1.2 \cdot \bar{t})$ with respect to an introduced hand coordinate system [7], edges are detected using a $7 \times 7$-Prewitt filter, see Fig 6 . Features are extracted as variances within blocks of size $24 \times 24$

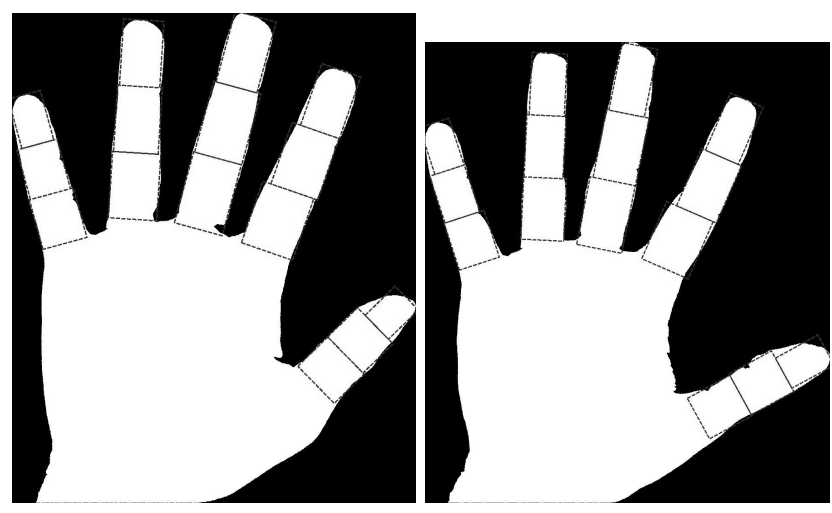

Fig. 5. Shape feature for 15-year-old child (left) vs. adult (right). 

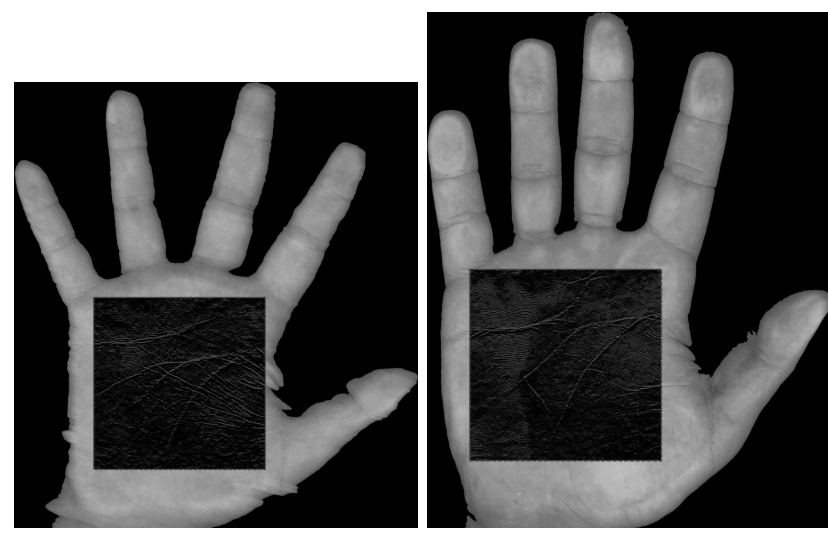

Fig. 6. Palmprint of 9-year-old girl (left) vs. adult (right).

TABLE II

COMPARISON OF KIDS AND ADULT AGE GROUPS WITH RESPECT TO EER (MEASURED IN PERCENT)

\begin{tabular}{|c|c|c|c|}
\hline Feature & Age 3-10 & Age 11-18 & Age 19+ \\
\hline \hline Thumb & 2.8 & 2.4 & 2.3 \\
\hline IndexFinger & 1.6 & 1.6 & 1.2 \\
\hline MiddleFinger & 2.5 & 1.2 & 0.6 \\
\hline RingFinger & 4.4 & 1.6 & 1.2 \\
\hline LittleFinger & 11.2 & 4.5 & 5.5 \\
\hline EThumb & 10.3 & 8.8 & 10.1 \\
\hline EIndexFinger & 6.8 & 8.2 & 7.1 \\
\hline EMiddleFinger & 4.5 & 4 & 2.3 \\
\hline ERingFinger & 4.8 & 4 & 3.3 \\
\hline ELittleFinger & 5.8 & 5.4 & 5.3 \\
\hline EPalm & 10 & 7.2 & 8.1 \\
\hline Palmprint & 5.6 & 5.3 & 3 \\
\hline Shape & 3.6 & 2 & 3.7 \\
\hline Geometry & 4 & 4.4 & 6.4 \\
\hline
\end{tabular}

pixels within a downscaled and normalised $300 \times 300$ version of the palm, as proposed in [11]. The Palmprint matcher computes a normalised squared Euclidian distance between feature vectors.

\section{EXPERIMENTS}

In order to assess performance differences between different child age groups (ages 3-10, 11-18) and an adult age group (19+), we compare verification accuracy by employing the following performance indicators with respect to crosscomparisons of templates within sets introduced in Sect. II: (a) equal error rate (EER), defined as the error where false match rate (FMR) and false non-match rate (FNMR) are equal (see Tab. II); and (b) receiver operating characteristics (ROC) plotting pairs of FMR and FNMR values for all possible thresholds. In order to get a more diversified picture of relative performance with respect to individual instances, we do not combine different fingers in our evaluation, like [2], but evaluate instances (fingers) and features individually.

\section{A. Impact of age on Minutiae}

Generally, age group 3-10 exhibits lower performance with respect to EER compared to the groups 11-18 and 19+, see Figs. 7-11. Performance differences between age 11-18 and age $19+$ are less pronounced, but still visible. Contributing factors for a degradation in performance were observed interaction issues, such as inconsistent finger placement, see Fig. 2. As can also be seen in this figure, due to the smaller fingerprint area in kids' hand images, the number of found minutiae is obviously reduced. When comparing differences between single instances, we find the following:

- Thumb: almost no significant differences in performance between all three age groups can be identified. This is plausible, since it already has bad performance compared to other fingers in age group 19+, accuracy differences to other fingers becomes less visible for kids.

- Index: little differences exist, slightly better performance of age 19+ especially for requested low FMR is observed. Since young children early develop coordination abilities of their index finger, we found rather good placements on the sensor, which contributes to better minutiae extraction and might explain, why there is less visible difference between age groups.

- Middle: age group 3-10 shows significantly worse performance compared to the other age groups. This might be caused by the size of the fingerprint region (depending on the finger length), which is critical for good performance.

- Ring: we found clearly different performance for all 3 age groups, i.e. the performance of the Ring finger minutiae feature increases up to adult age. A possible explanation of this behaviour is the region size mentioned before.

- Little: unlike the other Minutiae instances, we observe the best performance with age group 11-18 followed by age group 19+ and age 3-10. While the worse performance of age group 3-10 is easily explained by the smallest fingerprint size and weak coordination abilities as outlined before, the better performance of the youth age 11-18 group might be caused by less worn fingerprint texture compared to adults, who are less using this finger and sometimes have difficulties to achieve perfect sensor contact.

\section{B. Impact of age on Eigenfinger and Eigenpalm}

Age seems to have less impact on Eigenfingers as can be seen from Figs. 12-16. For some instances, age group $19+$ tends to deliver better results, which might generally be caused by the employed model (all Eigenfingers were calculated from a separate training set of people in this age group).

- Thumb: considering the rather high error rate of the Eigenfingers feature on the Thumb instance, performance differences are not very pronounced with a slight better behaviour of the age group 11-18. A possible reason may be given as follows: this instance is highly affected by different pose, while for young children the model might be less accurate, adults exhibit higher intra person variability.

- Index: ROC curves are quite close indicating no significant differences between age groups. 


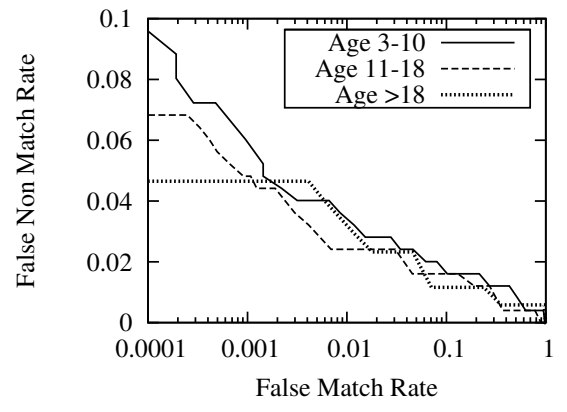

Fig. 7. ROC of Thumb (Minutiae).

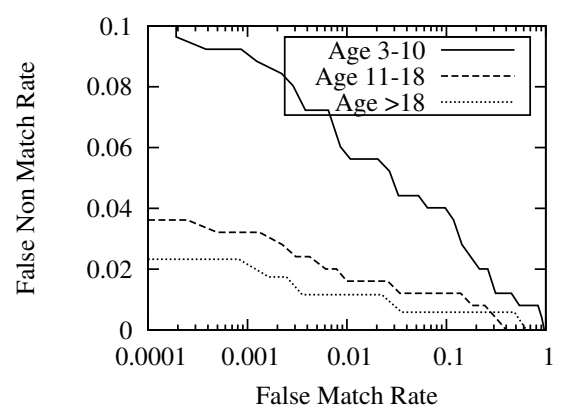

Fig. 10. ROC of Ring finger (Minutiae).

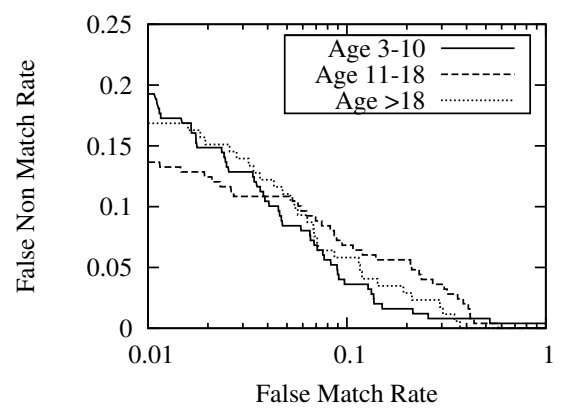

Fig. 13. ROC of Index finger (Eigenfinger).

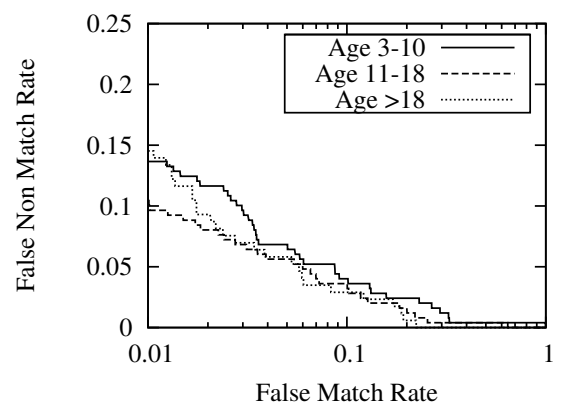

Fig. 16. ROC of Little finger (Eigenfinger).

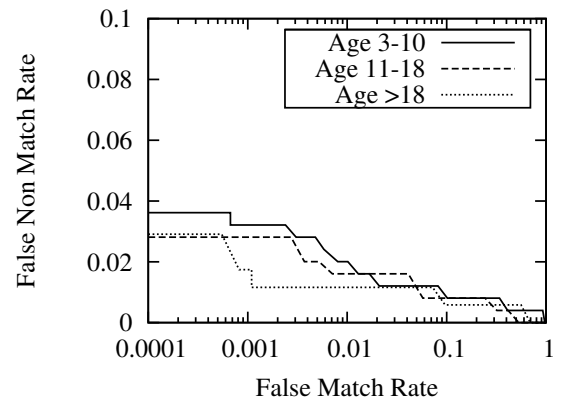

Fig. 8. ROC of Index finger (Minutiae).

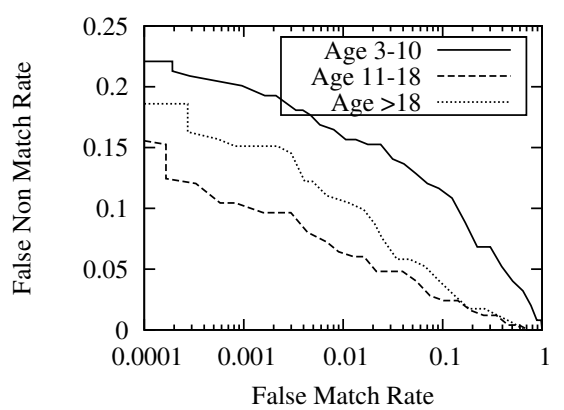

Fig. 11. ROC of Little finger (Minutiae).

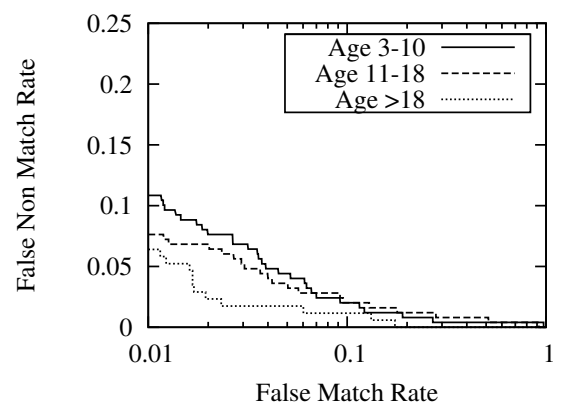

Fig. 14. ROC of Middle finger (Eigenfinger).

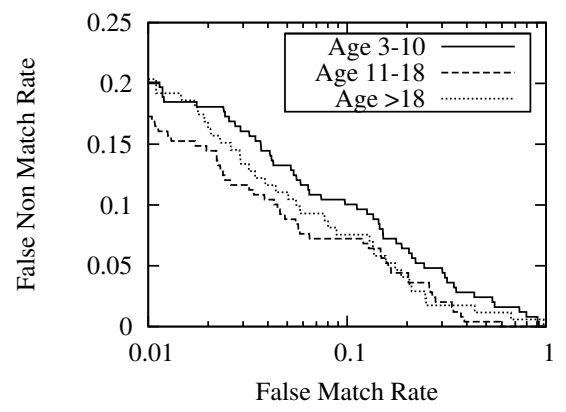

Fig. 17. ROC of Palm (Eigenpalm).

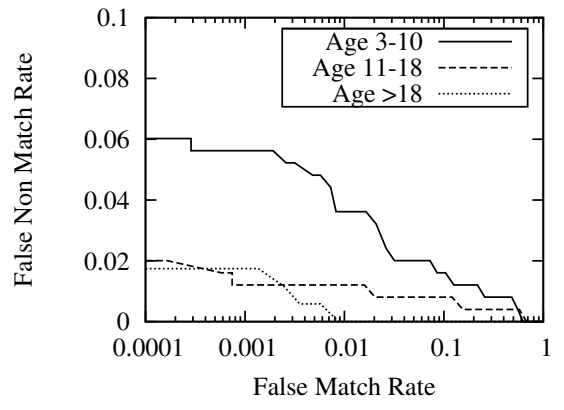

Fig. 9. ROC of Middle finger (Minutiae).

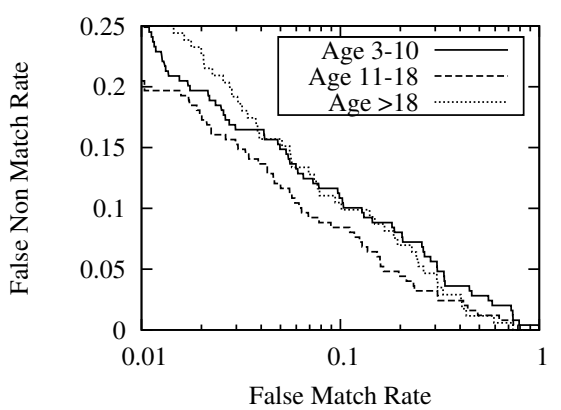

Fig. 12. ROC of Thumb (Eigenfinger).

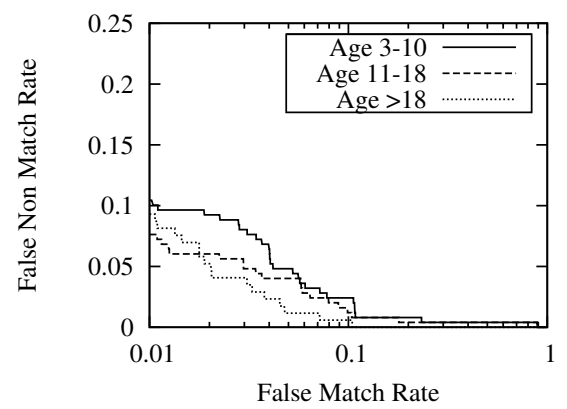

Fig. 15. ROC of Ring finger (Eigenfinger).

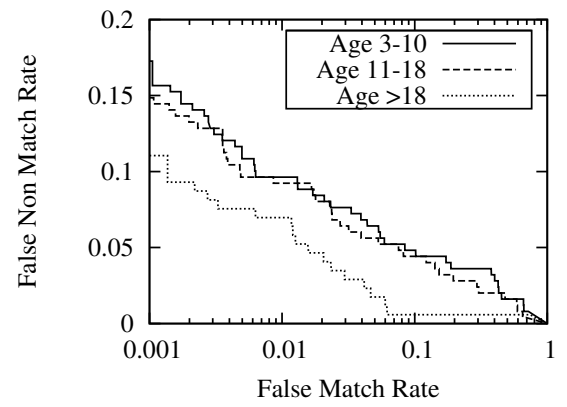

Fig. 18. ROC of Palm (Palmprint). 


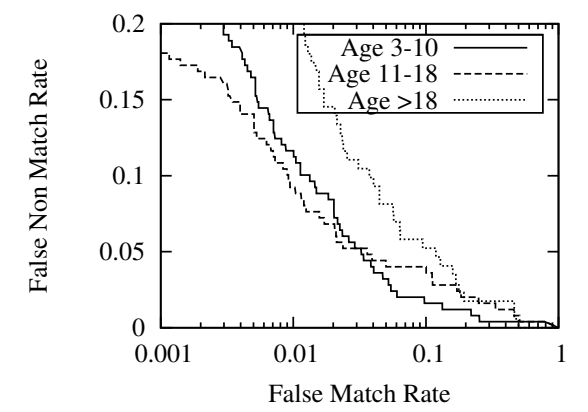

Fig. 19. ROC of Geometry.

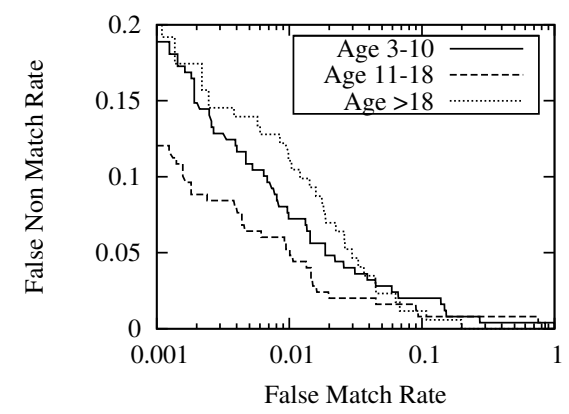

Fig. 20. ROC of Shape.
- Middle: age group 19+ shows better performance than other tested groups. As a reason for this behaviour we again refer to the argument, that the employed model was designed for this group. Middle fingers of children are much shorter, thus not well represented.

- Ring: similar performance of all age groups.

- Little: very little differences between age groups.

For the Eigenpalm feature, we found age group 11-18 outperforming age 19+ and age 3-10. In contrast to fingers, palm texture of persons belonging to age group 11-18 is expected to perform well, since it exhibits less wrinkles and creases, and thus lower intra-personal variance, at still being well-represented in the employed model.

\section{Impact of age on Palmprint}

Again, age group 19+ performs clearly better than other groups with the Palmprint feature. Employed edge detection methods are sensitive to the smoothness of the edges to be detected. Smaller palms cause smoother edges in the rescaled image (all images are rescaled to a specific resolution), i.e. the problem is an insufficient model.

\section{Impact of age on Shape}

Age group 11-18 is the best performing age group for Shape, followed by age 3-10 and age 19+. However, this is plausible since a rather high inter class variance for age group 11-18 due to varying growth effects, and thus higher accuracy can be expected.

\section{E. Impact of age on Geometry}

Age group 19+ performs worse than the other two age groups for Geometry. A plausible reason may be explained as follows: finger and segment length detection might be affected by wrinkles. Longer fingers tend to have more segmentation errors.

\section{CONCLUSIONS AND FUTURE WORKS}

We have examined verification performance of two different age groups of children $(3-10,11-18)$ with respect to Fingerprint, Eigenfinger, Palmprint and Digitprint biometrics and compared results with an adult age group (19+). Significantly worse performance of the youngest age group (3-10) was observed for the Middle, Ring and Little fingers in case of fingerprint (minutiae), and for the Palmprint feature.
An inverse behaviour (best performance of age group 3-10) was reported for Geometry. For the youth group (age 1118), differences to the other two groups are less pronounced, interestingly for Shape this feature clearly outperforms the other features. Adults (19+) are generally best-represented, with few exceptions. Furthermore, this work tried to identify possible explanations for the observed behaviour by visual inspection. Future topics of interest comprise the adaption of employed models (e.g. Eigenfingers) to better fit child data.

\section{REFERENCES}

[1] S. Modi and S. Elliott, "Impact of image quality on performance: Comparison of young and elderly fingerprints," in Proc. 6th International Conference on Recent Advances in Soft Computing (RASC), 2006, pp. $449-454$.

[2] S. Modi, S. Elliott, J. Whetsone, and H. Kim, "Impact of age groups on fingerprint recognition performance," in IEEE Workshop on Automatic Identification Advanced Technologies, 2007, pp. 19-23.

[3] D. Maltoni, D. Maio, A. Jain, and S. Prabhakar, Handbook of Fingerprint Recognition. New York, NY, USA: Springer Verlag, 2003.

[4] R. Rowe, U. Uludag, M. Demirkus, S. Parthasaradhi, and A. Jain, "A multispectral whole-hand biometric authentication system," in Proc. Biometrics Symposium, 2007.

[5] A. Uhl and P. Wild, "Personal recognition using single-sensor multimodal hand biometrics," in Image and Signal Processing. Proceedings of ICISP 2008, ser. LNCS, A. Elmoataz, O. Lezoray, F. Nouboud, and D. Mammass, Eds., vol. 5099. Springer Verlag, 2008, pp. 396-404.

[6] J. Aguilar-Fierrez, L. Munoz-Serrano, F. Alonso-Fernandez, and J. Ortega-Garcia, "On the effects of image quality degradation on minutiae- and ridge-based automatic fingerprint recognition," in Proceedings of the 39th Annual International Carnahan Conference on Security Technology, 2005, pp. 79-82.

[7] A. Uhl and P. Wild, "Single-sensor multi-instance fingerprint and eigenfinger recognition using (weighted) score combination methods," International Journal on Biometrics, vol. 1, no. 4, pp. 442-462, 2009.

[8] National Institute of Standards and Technology, "Fingerprint Image Software 2," 2006, http://fingerprint.nist.gov/NFIS.

[9] S. Ribaric and I. Fratric, "A biometric identification system based on eigenpalm and eigenfinger features," IEEE Transactions on Pattern Analysis and Machine Intelligence, vol. 27, no. 11, pp. 1698-1709, 2005.

[10] A. K. Jain, A. Ross, and S. Pankanti, "A prototype hand geometrybased verification system," in Proceedings of the 2nd International Conference on Audio- and Video-based Biometric Person Authentication (AVBPA'99), 1999, pp. 166-171.

[11] A. Kumar, D. Wong, H. Shen, and A. Jain, "Personal verification using palmprint and hand geometry biometric," in Proceedings of AVBPA, ser. LNCS, J. Kittler and M. Nixon, Eds., vol. 2688. Springer Verlag, 2003, pp. 668-678.

[12] K. Zuiderveld, "Contrast limited adaptive histogram equalization," in Graphics Gems IV, P. S. Heckbert, Ed. Morgan Kaufmann, 1994, pp. 474-485.

[13] M. Turk and A. Pentland, "Eigenfaces for recognition," Journal of Cognitive Neuroscience, vol. 3, no. 1, pp. 71-86, 1991. 